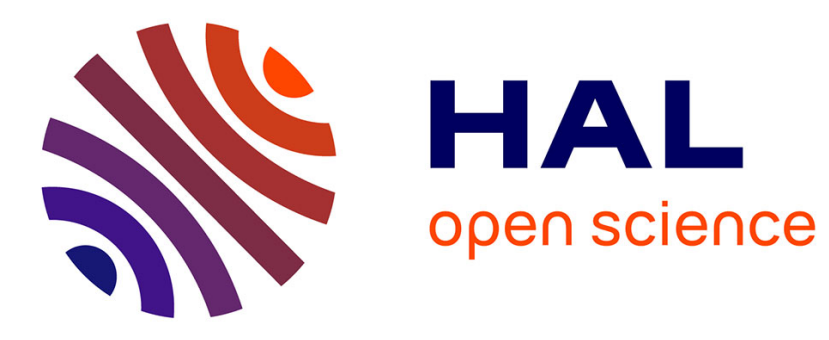

\title{
Multiphysics Modelling Applied to Refractory Behaviour in Severe Environments
}

Eric Blond, Tarek Merouki, Nicolas Schmitt, Emmanuel de Bilbao, Alain Gasser

\section{- To cite this version:}

Eric Blond, Tarek Merouki, Nicolas Schmitt, Emmanuel de Bilbao, Alain Gasser. Multiphysics Modelling Applied to Refractory Behaviour in Severe Environments. 13th International Ceramics Congress (CIMTEC, 2014), Jun 2014, Montecatini Terme, Italy. pp.301 - 309, 10.4028/www.scientific.net/AST.92.301 . hal-01716206

\section{HAL Id: hal-01716206 \\ https://hal.science/hal-01716206}

Submitted on 18 Feb 2019

HAL is a multi-disciplinary open access archive for the deposit and dissemination of scientific research documents, whether they are published or not. The documents may come from teaching and research institutions in France or abroad, or from public or private research centers.
L'archive ouverte pluridisciplinaire HAL, est destinée au dépôt et à la diffusion de documents scientifiques de niveau recherche, publiés ou non, émanant des établissements d'enseignement et de recherche français ou étrangers, des laboratoires publics ou privés. 


\title{
Multiphysics modelling applied to refractory behaviour in severe environments
}

\author{
BLOND Eric ${ }^{1 *}$, MEROUKI Tarek ${ }^{2}$, SCHMITT Nicolas ${ }^{3}$, de BILBAO \\ Emmanuel $^{4}$, GASSER Alain ${ }^{1, b}$ \\ ${ }^{1}$ Univ. Orléans, PRISME (EA4229), Polytech Orléans, 8 rue L. de Vinci, 45072 Orléans, France \\ ${ }^{2}$ Univ. of Versailles Saint-Quentin, LISV (EA4048), 78140 Vélisy, France \\ ${ }^{3}$ LMT Cachan, ENS de Cachan, 61 av. pdt Wilson, 94235 Cachan, France \\ 1eric.blond@univ-orleans.fr, ${ }^{2}$ tarek.merzouki@uvsq.fr, ${ }^{3}$ schmitt@Imt.ens-cachan.fr, \\ emmanuel.debilbao@univ-orleans.fr,
}

Keywords: refractory, multiphysics coupling, SiC oxidation,

\begin{abstract}
It is a common practice to design refractory linings with the help of thermal computations, thermochemistry analyses and strong workman know-how. Their mechanical design is often limited to simple thermo-elastic computations. Sometimes computations are refined considering non-linear mechanical behaviour, even if corrosion often induces additional chemical strain and strong change in service of the mechanical behaviour of the refractory. The aim of this presentation is to briefly recast the irreversible thermodynamic framework in order to underline the implications of some basic thermodynamic concepts in term of refractory behaviour modelling. Then, the use of these concepts to develop fully 3D finite element simulations accounting simultaneously for thermal, mechanical and chemistry phenomena will be illustrated on the particular case of SiC-based refractory. Comparison between long duration oxidation test at high temperature and model prediction allows the validation of the proposed approach. Then, an extension to the industrial case of refractory lining in Waste to Energy plant will be illustrated. The interest of taking into account the thermo-chemo-mechanical coupling effects is shown.
\end{abstract}

\section{Introduction}

Refractories are often subjected to extreme solicitations: high temperature gradient (imposed by the process), aggressive chemical environment (due to the process) and mechanical constraint (due to the design). Consequently, refractory degradations result from these combined solicitations. They induce a strong complexity in the design of large refractory lining structures which adds many engineering difficulties. Then, the degradation of refractory is a fully multi-physics problem. In addition as illustrated by figure 1, the different physics that play an important role in the degradation process do not take place at the same scale, so that degradation of refractory is also a a multi-scale problem. Thanks to specific studies of corrosion [1], phase change [2] and thermomechanics [3], problems occurred in fire industrial processes, and solved by specialists from different scientific fields (at different scales) permitted important progresses of the life-time of refractory linings. Although specialists acknowledge that pooling their resources could help to explore new ways to improve refractories, cross-studies are less common. In particular, thanks to developments of new experimental and numerical tools in different fields a better knowledge of the causes of degradations due to the combination of multi-physical phenomena seems to be now possible.

Nowadays, development of finite elements analysis (FEA) and of thermochemistry software [4] permits to foresee lining simulations taking into account both thermo-mechanics analysis (i.e. structural effects in sense of geometry and mechanical behaviour) and thermo-chemistry analysis (in 
sense of corrosion or phase change inducing mechanical effect). Such new type of coupled computations corresponds to the dashed black line on Fig. 1. The two main limits for the achievement of such computations are the definition of the coupling terms and the adaptation/development of numerical tools. While the second key point is on the point of being solved from two different ways (coupling of existent softwares or development of dedicated fullycoupled software) the first point is still be open: how writing the coupling(s) term(s) without too many un-identifiable parameters while maintaining a physical meaningful description of the reality? Even there may be no uniqueness of the answer, the extended irreversible thermodynamics provides an comprehensive and precise framework with some good guidelines to do it.

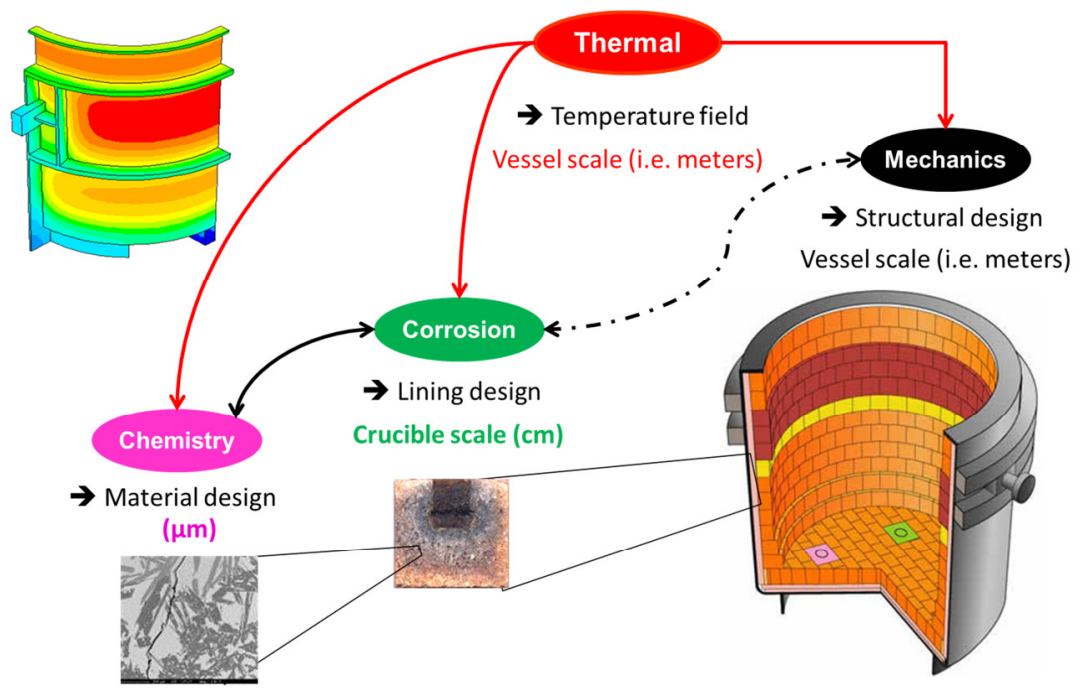

Figure 1: Graphical sum up of the design of refractory lining: a fully multi-physic and multiscale problem.

The aim of this paper is to briefly recast the irreversible thermodynamics framework in order to underline the implications of some basic thermodynamic concepts in term of refractory behaviour modelling to develop a fully 3D numerical simulation accounting for thermal, mechanics and chemistry with the goal to take into account the effect of corrosion. To illustrate the concepts and highlight the relevance of such approach, the particular case of the degradation of oxide bonded SiC-based refractory used in Waste-To-Energy facilities (WTE) serves as a guideline.

\section{Presentation of the guide line: degradation of the lining of Waste-To-Energy plant}

In WTE, a refractory lining protect the metallic water wall, which allows a maximum recovery of heat from hot ashes and gases resulting from the burning of waste, against corrosion at high temperature. The thickness of the refractory lining is small (approximately $60 \mathrm{~mm}$ ) to facilitate the heat transfer. On one side, the refractory lining is in contact with the metallic water wall at a temperature of approximately $250^{\circ} \mathrm{C}$. On the other side it is in contact with smoke and ashes. The temperature in the combustion chamber at the bottom level in the hot flame is close to $1200^{\circ} \mathrm{C}$. About $17 \mathrm{~m}$ higher, at the top of the chamber, it falls down to around $500^{\circ} \mathrm{C}$.

It is difficult to have a detailed and in-depth understanding of the environment conditions in the combustion chamber because the nature and content of fumes and ashes depend on the waste burned and the incinerator process. Consequently numerous mechanisms of corrosion may be involved in the chemical degradation of the $\mathrm{SiC}$-based refractory. Observations made on worn parts of the refractory lining have shown that several corrosion mechanisms take place with the time [5]. | For example, post-mortem analyses on worn oxide bonded $\mathrm{SiC}_{-}-$based tiles have shown that molten salts react with $\mathrm{SiC}$ aggregates and matrix of the material and form para-wollastonite around $\mathrm{SiC}$ 
grains and in the porosity, near the hot face of refractory tiles. Other phases such as cristobalite and microline are also formed down to the core of tiles. One scenario of corrosion proposed by Prigent [5] is the following. It starts with the migration of oxygen molecules $\mathrm{O}_{2}$ into the refractory through the porosity. The gas $\mathrm{O}_{2}$ react with $\mathrm{SiC}$-grain to form $\mathrm{SiO}_{2}$ layer surrounding the sound part of $\mathrm{SiC}$ grain. Then, oxidation products may react with some other fumes components (like $\mathrm{Ca}$ ) to form other products of alkaline corrosion (like $\mathrm{CaSiO}_{3}$ ).

At macroscopic scale, the degradation of the refractory layer depends on both the material and the geometry of tiles. But progressive swelling of the material during service is a common mechanism observed in different plants with the result that stresses are introduced that can locally lead to rupture. An illustration of a deformed tile is shown Fig.2.

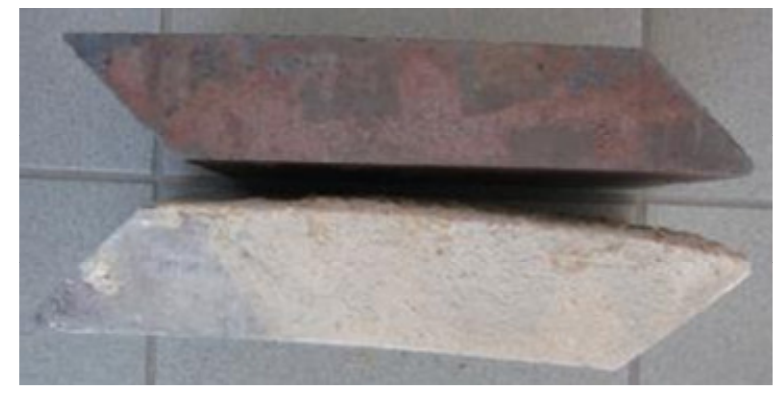

Figure 2: Worn (at the bottom) and new (at the top) tiles.

Hereafter, attention will be exclusively focused on the oxidation of $\mathrm{SiC}$. This choice is justified by the fact that it is the first step of the degradation mechanism and it often (close to always) participates to the degradation of $\mathrm{SiC}$-based refractory structures. Furthermore, for sack of simplicity, it is easier to illustrate the thermodynamics framework that allows to couple thermochemistry and thermomechanics with only one chemical reaction.

\section{Essential basic concept of thermodynamics and macroscopic modelling}

The first important step for macroscopic modelling is to define a representative elementary volume (REV) which is large enough regard to the size of the heterogeneities of the material. This is required to ensure that averaged values of state variables (strain, stress, ...) over this volume are relevant to describe the macroscopic behaviour i.e., a behaviour which do not depend on the size of the elementary volume of the material. This definition of the REV has to be understood regard to homogenization technique that permits to define an equivalent homogeneous media from the strain energy point of view [6]. The material considered hereafter is a commercial $\mathrm{SiC}$-based refractory, having a particle size distribution ranging from $20 \mu \mathrm{m}$ to $3 \mathrm{~mm}$ and containing approximately 85 wt.\% of $\mathrm{SiC}$-grains. Its porosity is equal to $17 \%$. Few large pores are observed at the millimetre scale but the connected porosity (small pores, microcracks) mainly exists at the micrometre scale, so that most of the oxidation effects takes place in the matrix phase. For this case, the REV is close to one $\mathrm{cm}^{3}$. At this scale, this material can be viewed as an open, reactive, multiphasic, heterogeneous, deformable medium exchanging mass, heat and work with its environment. It can be modelled as a continuous homogeneous porous multi-phasic medium. In this medium, the different phases (initial skeleton, void, gas, liquid, solid product of reaction) are assumed to be superimposed at each point. To ensure a representative global behaviour it is assumed that, at each point of the medium, the macroscopic properties are defined as volumic average of local properties $[7,8]$.

Once the REV has been defined it is essential to well identify the state variables to describe the behaviour of the system. As it is not so obvious to do it when dealing with multi-physical problems, the use of a coupling scheme is recommended [9]. This approach simplifies drawing of the physics in presence and their interactions and permits to not forget a coupling effect. Before to choose the state variables, there is an interest to remember the Callen's remark [7]: "The 
equilibrium is assumed to be characterized completely by the extensive independent parameters. It follows that the properties of a system must be independent of the past history". Often in the refractory community, appropriate state variables have not been well identified because there is often (always ?) observed an history effect. Indeed, as an illustration, many different effects more or less linked to chemistry and phase change are hidden in the temperature dependence of properties like Young's modulus or coefficient of thermal expansion. Then, to develop a multi-physical model, this habit should be discarded. Indeed, as the equilibrium state is defined by the values of state variables that minimize the internal energy (thermostatic) or maximize entropy (irreversible process), a bad initial definition of the state variable will ruin the chance to obtain physically meaningful prediction. A proper identification of the set of state variables characterizing the system requires taking (long) time for rigorously analysis of available data (measurement, observation, know-how) and a careful identification of the phenomena that play a role and their interactions. In the application treated here, the physics taken into account are: heat transfer, mechanics, chemistry (i.e. $\mathrm{SiC}$ oxidation) and diffusion (i.e. oxygen flux through the gas into the porosity). The good state variables should be the temperature (easier to use than the entropy, even if it is not an extensive variable), the strain (for the mechanical state), the oxygen partial pressure in fumes/gas (playing a role in diffusion) and the mass fraction of $\mathrm{SiC}$ or the mass fraction of $\mathrm{SiO}_{2}$ or the extent of oxidation. If the chemical content of $\mathrm{SiC}$ or $\mathrm{SiO}_{2}$ is taken as state variable, it requires the quantification of the local content of $\mathrm{SiC}$ at each point. The oxidation extent instead of the $\mathrm{SiO}_{2}$ mass fraction or the $\mathrm{SiC}$ content seems to be a better state variable because initially it is null at each point and it reduces the number of variable to compute (one instead of two that, are not independent). Moreover, from the chemical modelling point of view, the extent will be helpful to introduce the kinetics of oxidation. Finally, the local state of the system is characterized by four state variables: temperature $(T)$, strain $(\varepsilon)$, oxygen partial pressure $(p)$ and the extent of oxidation $(\xi)$.

Once the state variables are defined, it is possible to write the local balance laws of the system. It is essential to be aware that balance laws only concerne extensive variables, like mass, momentum, energy and entropy. Heat is not an extensive variable, the so-called "heat equation" results from the entropy balance and the energy balance, accounting for evolution and exchange laws $[7,8]$. Then, the definition of the fully coupled heat equation requires a huge background to well define the appropriate pseudo-potential of dissipation [10]. For refractory lining, a simple and general form for the heat equation is [10]:

$$
\rho C_{\varepsilon} \frac{d T}{d t}=-\operatorname{div}\left(\overrightarrow{\boldsymbol{q}}+\sum_{i} \frac{h_{i}}{M_{i}} \overrightarrow{\boldsymbol{J}}_{i}\right)-\rho \sum_{r} \dot{\xi}_{r} \Delta_{r} H
$$

where $\rho$ denotes the average bulk density of the material and $C_{\varepsilon}$ the specific heat for constant volume. Index $i$ denotes the species, $\vec{J}_{i}$ the mass flux of species $i, M_{i}$ the molar mass for $i, \dot{\xi}_{r}$ the extent rate of the chemical reaction $r$ and $\Delta_{r} H$ the enthalpy of reaction $r$. In industrial vessel, as the temperature gradient is often high and imposed by the process, the source term due to chemical reaction and the convective heat flow (in the local heat equation (1) it correspond to the heat transported by gas in the porosity of the refractory : $\sum_{i} \frac{h_{i}}{M_{i}} \vec{J}_{i}$ ) can be neglected.

One important contribution of the chemical reactions concerns the mass balance equation, that writtes :

$$
\rho \dot{x}_{i}=-\operatorname{div} \overrightarrow{\boldsymbol{J}}_{i}+\sum_{r} v_{i r} M_{i} \dot{\xi}_{r}
$$

where $x_{i}$ denotes the mass ratio of species $i$ and $v_{i r}$ the stoechiometric coefficient for species $i$ in reaction $r$. The definition of the source term in the mass balance requires to know the kinetics of 
reaction which still being a challenge in lot of practical cases. Here, for the case of oxidation of oxide bonded $\mathrm{SiC}$ based refractory, the extent rate can be defined by [10]:

$$
\dot{\xi}=\frac{1}{2} \frac{k_{p}}{\xi}\left(\frac{\rho x_{O 2}}{M_{O 2}}\right)^{2}\left(1-\frac{Q_{p}}{K_{p}}\right)^{2}
$$

where $k_{p}$ is the rate constant following an Arrhenius law, $Q_{p}$ the mass action value at considered time and $K_{p}$ the equilibrium constant. It is worth nothing that the equilibrium constant is equal to the mass action value at the equilibrium. The definition of the extent rate must be equal to 0 at equilibrium and the product with the affinity must always ensure a positive entropy rate (see $[7,8,10])$.

In the WTE case the flux of each species is assumed resulting from diffusion through the gas in the porosity of the material. At local scale, some of them may react with the solid at the surface of pores and microcracks, creating new solid and gaseous products. The deposit of an oxide layer progressively clogs up the porosity leading to a reduction of the diffusivity with the extent of oxidation. Due to lack of information, to account for this reduction, it is assumed that the porosity decreases linearly with the extent of the reaction and the diffusivity changes linearly with porosity [11]. This evolution corresponds to the observed densification of the refractory $[5,11]$.

The thermo-mechanical "behaviour" of the material usually encompasses the state laws and the evolution laws of the material. In the study case, the behaviour is described by a linear elastic law and thermal strain $\left(\boldsymbol{\varepsilon}_{T}\right)$ with a constant coefficient of thermal expansion is considered. The swelling induced by corrosion (dissipative mechanisms) can be described by an evolution law of the mechanical state variable (strain, $\boldsymbol{\varepsilon}_{c}$ ) which is depending on the chemical state variable (extent). Entropy rate is the key to establish such coupling terms respecting the energy conversion rule [7]. Assuming small strain, strain partition can be considered. The total strain tensor $(\varepsilon)$ becomes the sum of the thermal strain, the mechanical strain $\left(\boldsymbol{\varepsilon}_{e}\right)$ and the corrosion induced strain $\boldsymbol{\varepsilon}_{c}$. Assuming a quadratic thermodynamic pseudo-potential, a linear dependence between chemical strain and the extent can be established $[3,4]$ :

$\boldsymbol{\varepsilon}_{c}=\beta \cdot \xi \cdot \boldsymbol{I}$

where $\boldsymbol{I}$ is the second rank identity tensor and $\beta$ is the coefficient of chemical strain or "chemical expansion". In the study case, the identification of $\beta$ has been done thanks to correlation between thermogravimetrical analyses (TGA) and isothermal dilatometry [11]. Indeed, assuming a homogeneous oxidation in the dense samples during dilatometry, after the same duration, the extent in the dilatometric sample should be the same that in TGA sample. The validation of the chemical expansion term has been done thanks to a dedicated test [11] and the identification of elastic parameters has been done using three-point bend test and ultrasonic measurements [12].

\section{Computational procedure}

The reactive transport equation (Eq. 1), including the oxidation extent evolution (Eq. 3), has been implemented in the software ABAQUS thanks to a UMATHT subroutine. To do this, equations are re-written in function of partial pressure of gases in the pores [11]. The chemical strain function of extent of oxidation (Eq. 4) is implemented in ABAQUS via the UEXPAN subroutine. As often for such unidirectional couplings, the uncoupled heat transfer is solved first. Then, the calculated temperature field is prescribed for the reactive transport computation. Finally, the temperature and oxidation extent fields are prescribed as a part of the loading in the mechanical computation. 


\section{Validation test}

In order to validate the previous model, a numerical simulation was performed of an experimental oxidation test of a $\mathrm{SiC}$-based castable cylinder subjected to an axial thermal gradient from room temperature to $1100^{\circ} \mathrm{C}$ for duration of 56 days. The main goals were: 1) validating the expansion law (Eq. 4) and the identification of the chemical expansion coefficient $\beta$; 2) validating the temperature effect on oxidation extent. Experimental set-up, results and data used for the computation have been already presented in [11].

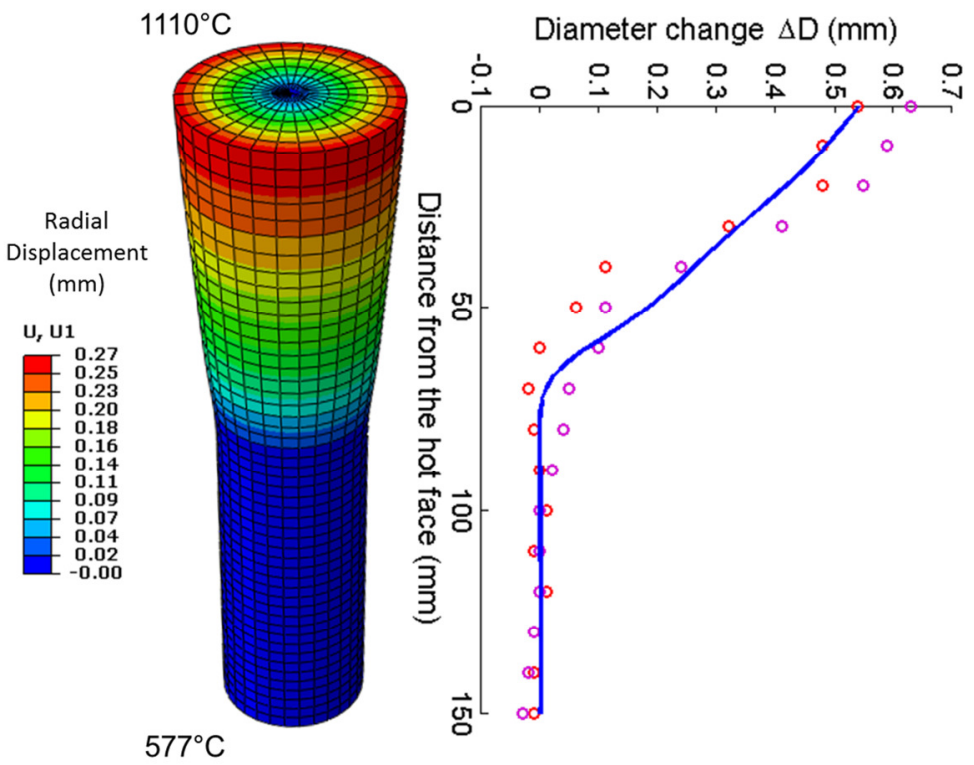

Figure 3: Experimental results and model prediction for long term oxidation under thermal gradient. The blue curve represents numerical results, while red and violet points, are experimental measurements along two perpendicular diameters.

The main results are summarised on Fig. 3. The variation in diameter all along the cylinder predicted by the numerical simulation and obtained experimentally after 56 days are plotted in the graph. At the left, the final shape, the final radial displacement and the extrema of temperatures during oxidation are represented. The discrepancy is lower than $20 \%$ for a distance from the hot face lower than $40 \mathrm{~mm}$. In the 40 to $80 \mathrm{~mm}$ range, the macroscopic strain is smaller and the difference too. The model gives an appropriate description of the physical phenomena since a good agreement between the experimental and numerical results can be observed. The combined effect of thermal gradient and oxidation induces a higher swelling at the hot face after cool down. It is firstly due to the thermal activation of oxidation and, secondary, it is linked to the slowdown of the oxygen transport induced by the matrix densification.

\section{Thermo-chemo-mechanical computation: a tool for lining design}

In order to show the potential of prediction of in-service behaviour of refractory systems with such a model, two geometries of lining tiles are have been modelled, corresponding to tiles A and B, respectively (Fig. 4). Tile A is placed on its handle, while tile B is tied on its top hole using mortar. For numerical convenience only the half part of the tiles have been modelled. The binding between water-wall and refractory lining is the main difference between both designs: hang up free (A) or interlock (B). Geometries of tiles correspond to real ones but material data used are those of a SiC based castable (CV85 from Calderys) so that these examples do not reproduce real industrial cases. The goal of these computations is to illustrate the interest of such approach, not to resolve a particular problem. 
For the thermal computations, convective heat flux was applied between refractory tiles and smoke and between pipe and steam with thermal exchange coefficients equal to $300 \mathrm{~W} \cdot \mathrm{m}^{2} \cdot \mathrm{K}^{-1}$ and $1000 \mathrm{~W} \cdot \mathrm{m}^{2} \cdot \mathrm{K}^{-1}$ respectively. The steam temperature was equal to $250^{\circ} \mathrm{C}$, and the smokes temperature equal to $1050^{\circ} \mathrm{C}$. A thermal resistance was introduced between pipe and tile defined as the thickness of the air layer divided by the thermal conductivity of air.

In the thickness of the tiles, the steady state temperature field was quite similar for both geometries. The small local difference $\left(14^{\circ} \mathrm{C}\right)$ was due to the hang up device which induces a higher thermal resistance for tile A and so localises the "hot point" just in front of the hang up on the hot face.

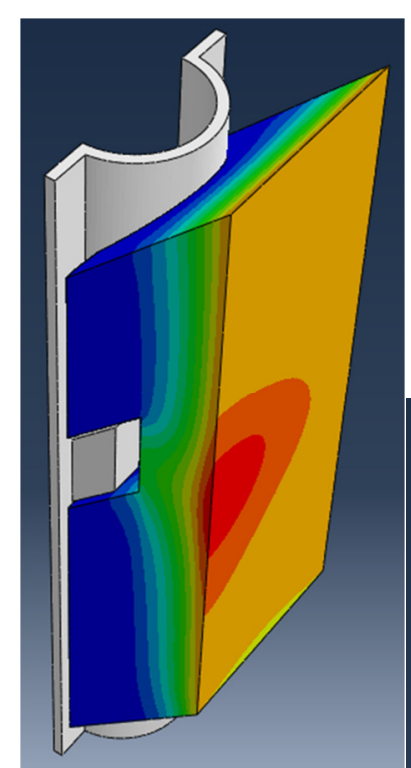

a) Tile B

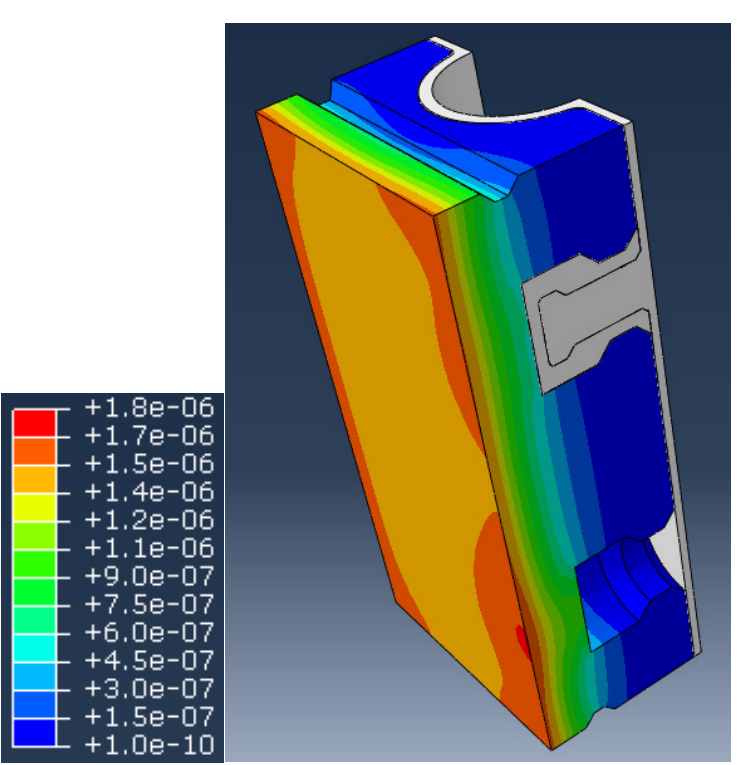

b) Tile A

Fig. 4. Effect of tile geometry on oxidation extent field $\left(\mathrm{mol} . \mathrm{mm}^{-3}\right)$ after one year in service for a $\mathrm{SiC}$-based castable assuming steady state temperature field.

For the simulation of the reactive transport, the initial partial pressure of oxygen in the lining was equal to that in the air. In service, a partial pressure of oxygen of $0.08 \mathrm{~atm}$ was imposed on the hot face, according to industrial measurements in fumes. At the cold face, the partial pressure of oxygen was imposed equal to zero because of the corrosion of the metallic pipe observed on real water wall of WTE facilities.

Computations confirm that the heterogeneous temperature field induces a heterogeneous oxidation: a high level of oxidation exists at the hot face and a low variation in the rest of the lining (Fig. 4). Design A shows a localized high extent pick while design B exhibits a more homogeneous oxidation but with a higher average extent. These differences directly result from the temperature field. Assuming a constant steady-state temperature field during one year, design A reaches the highest corrosion extent (localized in front of the hang up device) of the two designs.

It is essential to underline that a small variation of the stationary temperature field changes notably the state of corrosion after a long duration because the extent of oxidation is sensitive to the integration of the temperature on the duration.

For the simulation of the mechanical behaviour, the boiler tubes are clamped. A contact with friction is assumed between tiles and pipes. For design B, anchor and tile were pasted together by a mortar. Gravity force is taken into account and the mechanical behaviour of the SiC-based refractory is assumed, linear and elastic. This last assumption can lead to overestimation of the mechanical stresses that may be relaxed by local plasticity, creep and damage mechanisms. There is 
no difficulty in refining the mechanical behaviour in a next future. However, this refinement will not modify the expression of the strain induced by corrosion.

Figure 5 compares in tile with design A the vertical component of the stress tensor when the chemical strain is taken into account or not in the simulations. It is important to emphasize that the stress fields in the two analyses are totally different in term of localization and stress level. It is worth nothing that the simulations performed with thermo-elasticity material behaviour underestimates sensitively the stress when compared with simulation accounting for thermo-chemoelasticity. This result clearly shows that the elastic assumption should not be considered as the worst case. The field of chemical strain is highly heterogeneous because the oxidation kinetics is strongly linked to the temperature field. Discrepancies in localisation and level of stresses are consequence of the chemical strain induced by oxidation: the localisation of oxidation leads to a strong redistribution of stress in the structure and so will involve damage in area that thermo-elastic model cannot predict. This additive strain produces a bi-axial stress state in plane parallel to the hot face.

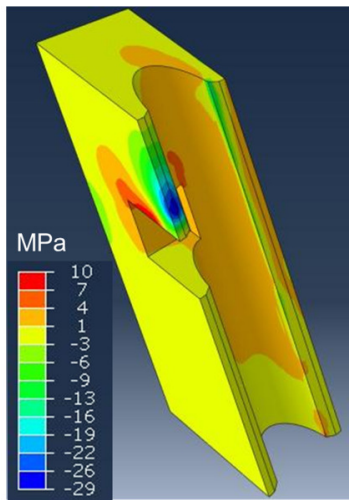

a) thermo-elasticity

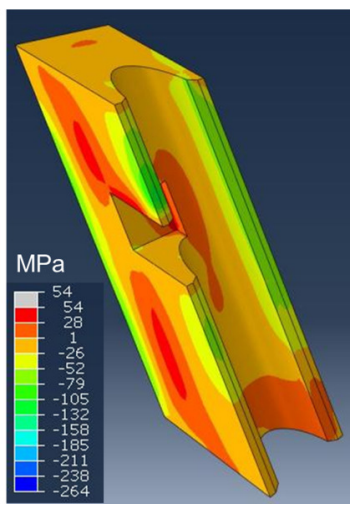

b) thermo-chemo-elasticity

Fig. 5. Vertical component of the stress tensor after one year in service for tile A

It is interesting to note that the localisation of maximal stresses predicted by the thermo-chemoelasticity computation is in quite good agreement with the crack pattern observed on (real) tile A (with another $\mathrm{SiC}$-based refractory material) after a long time spent in WTE (Fig. 6). The difference in material (i.e. binder, $\mathrm{SiC}$ content) pleads to conclude that the localisation of the crack is driven by the geometry which is of major importance for the temperature field and so for the extent field. Such result from simulation illustrates how thermo-chemo-mechanic model could help to better anticipate the effect of the coupling of geometry and corrosion in term of mechanical reliability. Moreover, as the chemical strain grows up with oxidation extent, oxidation kinetics drives the stress-time curve. However, the relationship is not linear and strongly depends on the temperature and geometry (i.e. stress concentration effect).

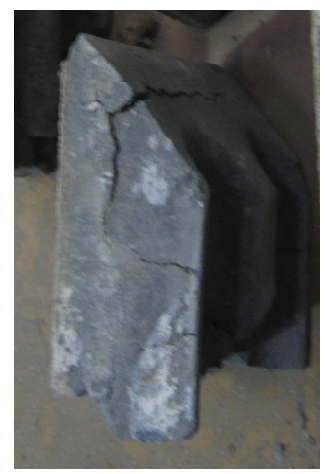

Fig. 6. Crack pattern on the top of post-mortem tile A 


\section{Conclusion}

The thermodynamic framework briefly recast here allows building analytical and numerical tools which are able to reproduce complex effects of thermo-chemo-mechanical coupling such as swelling induced by corrosion for example. One important difficulty to overcome to develop numerical simulations is the choice of the appropriate state variables and the identification of the coupling terms between physics of the model. Both stages require taking time for a fine analyse of mechanisms involved in order to identify clearly the underlying physics and their strictly necessary description to permit the building of the constitutive equations and the identification of the parameters.

The results presented here show that the thermo-chemo-elasticity permits to anticipate both the effects of oxidation and geometry on mechanical stress. Then, the crack patterns observed on real $\mathrm{SiC}$-based refractoy tiles from WTE plant could be now anticipate. Such computations show that it becomes possible to refine the prediction of the stress localisation accounting for corrosion, and so to envisage in a next future to obtain reasonable lifespan prediction and improve the design of refractory linings.

\section{References}

[1] Lee W. E. and Moore R.E., Evolution of in-situe refractories in the $20^{\text {th }}$ century, J. Am. Ceram. Soc., 81[6], 1381-1410, (1998)

[2] Schmitt N. et al., Coupling between kinetics of dehydration, physical and mechanical behaviour for high alumina castable, Cem. \& Conc. Res., 30 [10], p. 1597-1608, (2000)

[3] K. Andreev et al, "Thermo-mechanical behaviour of the refractory lining of a BOF converter-A numerical study," UNITECR 03, Osaka, Japan, p. 564-567 (2003)

[4] C. W. Bale et al., FactSage thermochemical software and databases, Calphad, 26 [2], p. 189-228 (2002)

[5]P. Prigent, et. al, Corrosion of oxide bonded silicon carbide refractories by molten salts in solid waste-to-energy facilities, Ceramics International 38 p. 5643-5649, (2012)

[6]Bornert, M., Bretheau, T., Gilormini, P.,.Homogénéisation en mécanique des matériaux, Vol. 1 : Matériaux aléatoires élastiques et milieux périodiques, (in French) Hermes (2001)

[7]H. B. Callen, Thermodynamics, Wiley \& Sons, (1960)

[8] O. Coussy, Mechanics and Physics of Porous Solids, Wiley \& Sons, (2010)

[9] E. Blond et al., Effect of slag impregnation on thermal degradations in refractories, J. Am. Ceram. Soc. 90 [1] p.154-162, (2007)

[10] J. Lemaitre and J.-L. Chaboche, Mechanics of solid materials, Cambridge University Press, (1990)

[11] T. Merzouki et al., Modelling of the swelling induced by oxidation in SiC-based refractory castables, Mechanics of Materials, 68, January, p. 253-266 (2014)

[12] E. De Bilbao et al., A new method to determine Young's modulus of refractory, Interceram, 59 [1] p.34-38 (2010) 